\title{
Cerebrospinal fluid pulse pressure and intracranial volume-pressure relationships
}

\author{
C. J . J.A V EZAAT, J.H.M.VA N E I N D H O V E N, \\ A N D D. J. WY PER
}

From the Departments of Neurosurgery and Electro-neurology, Academic Hospital RotterdamDijkzigt, Erasmus University, Rotterdam, the Netherlands, and the Department of Clinical Physics, Institute of Neurological Sciences, Glasgow

S U M MARY In six anaesthetised and ventilated dogs the CSF pulse pressure was compared with the volume-pressure response (VPR) during continuous inflation of an extradural balloon. Both pulse pressure and VPR increased linearly with the ventricular fluid pressure (VFP) up to a mean VFP of $60 \mathrm{mmHg}$. At this pressure a breakpoint occurred above which the CSF pulse pressure showed a steeper linear increase, while the VPR remained constant. It is suggested that the breakpoint is related to failure of autoregulation, and that in non-autoregulating patients the CSF pulse pressure is a better parameter of the clinical state than the VPR.

The rapid fluctuations in the cerebrospinal fluid (CSF) pressure synchronous with the heart beat (CSF pulse) have for a long time excited the interest of numerous workers. This interest concerns the origin, site of transfer, configuration, and magnitude of the pulse as well as its role in the pathogenesis and diagnosis of intracranial disease. The subject of the present study is the amplitude of the CSF pulse wave, hereafter termed the CSF pulse pressure. Table 1 contains a list of definitions of abbreviations and symbols used.

It is an old observation that the CSF pulse pressure increases concomitantly with the intracranial pressure (ICP) (Leyden, 1866; Mosso, 1881). This phenomenon can be explained by the exponential nature of the relationship between volume and pressure within the craniospinal compartment (Marmarou et al., 1975). With rising ICP, rapid intracranial volume changes such as those produced by the transient increase in cerebral blood volume (CBV) during each cardiac cycle will result in increasingly larger pressure changes (Fig. 1). From this concept the question arises whether the CSF pulse pressure can be used as a diagnostic tool to assess the intracranial volumepressure relationships. All hitherto described volume-pressure parameters require volume ad-

\footnotetext{
Address for reprint requests: Dr C. J. J. Avezaat, Department of Neurosurgery, Academisch Ziekenhuis Rotterdam-Dijkzigt, Erasmus University, Dr Molewaterplein 40, Rotterdam 3002, the Netherlands. Accepted 7 February 1979
}

Table 1 Definitions of abbreviations and symbols

\begin{tabular}{ll}
\hline $\begin{array}{l}\text { Abbreviation/ } \\
\text { symbol }\end{array}$ & Definition \\
\hline ICP & Intracranial pressure \\
VFP & Ventricular fluid pressure \\
CMP & Cisterna magna pressure \\
SAP & Systemic arterial pressure \\
$\Delta$ SAP & Systemic arterial pulse pressure \\
CPP & Cerebral perfusion pressure \\
Peq & Equilibrium pressure, physiological steady state ICP \\
$\Delta$ P & CSF pulse pressure or volume-pressure response \\
V & Change in total volume of the craniospinal \\
& compartment \\
Veq & Equilibrium volume of the craniospinal compartment \\
$\Delta$ V & at which ICP=Peq \\
& Injected volume during a volume-pressure test or the \\
CBV & transient increase in CBV per cardiac cycle \\
VPT & Cerebral blood volume \\
VPR & Volume-pressure test \\
E & Volume-pressure response \\
E & Elastance of the craniospinal compartment \\
PVI & Elastance coefficient \\
CBF & Pressure-volume index \\
CVR & Cerebral blood flow \\
HR & Cerebral vascular resistance \\
\hline & Heart rate \\
\hline
\end{tabular}

ditions to the CSF compartment (Shulman and Marmarou, 1971; Miller and Garibi, 1972; Löfgren et al., 1973). The most widely used parameter clinically is the volume-pressure response (VPR) which has been defined as the immediate change in ICP resulting from a uniform change in ventricular CSF volume (volume-pressure test; Miller and Garibi, 1972). The VPR is a measure of the intracranial elastance-that is, the instantaneous 


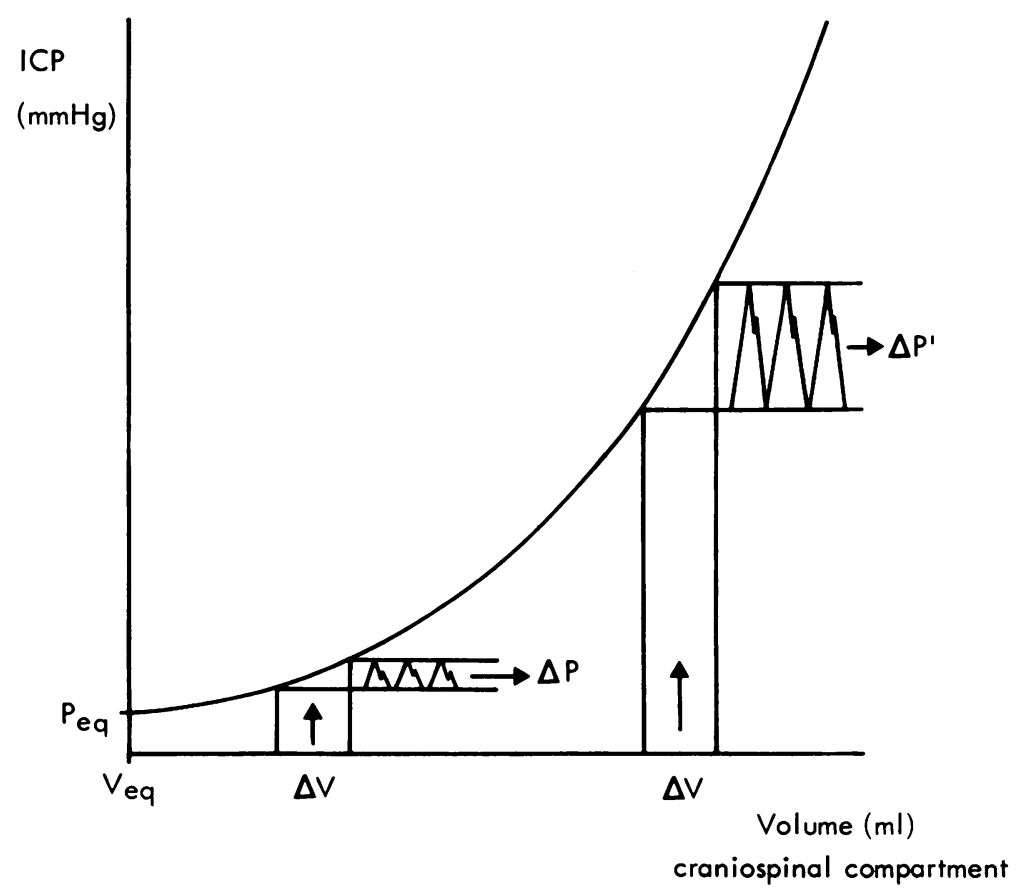

Fig. 1 The CSF pulse pressure is a pressure response $(\Delta P)$ to the transient increase in intracranial blood volume during a cardiac cycle $(\Delta V)$. The exponential shape of the intracranial volume-pressure curve explains why the CSF pulse pressure increases with rising intracranial pressure (ICP). Abbreviations defined in Table 1.

slope $(\mathrm{dP} / \mathrm{dV})$ of the exponential volume-pressure curve. The VPR has been claimed to show a better correlation with the clinical state of the patient than the magnitude of ICP alone (Miller et al., 1973; Miller and Pickard, 1974). However, some objections against the volume-pressure test (VPT) can be raised. The test increases infection hazards and cannot be used in epidural ICP measurements. In clinical practice the VPR shows rather a wide variability necessitating the use of multiple tests. Pressure waves may be provoked, while the alternative, aspiration of CSF, may lead to blockage of the ventricular catheter.

These disadvantages led us to devise a new method of evaluating intracranial volume-pressure relationships by using the CSF pulse pressure in relation to the ICP as an index (Avezaat et al., 1976), and others have been working along the same lines (Castel and Cohadon, 1976; Nornes et al., 1977; Szewczykowski et al., 1977). This method was based on the analogy between the CSF pulse pressure and the VPR, both being pressure responses to rapid changes in intracranial volume. In a group of patients the CSF pulse pressure was found to be related linearly to the ICP. However, since the CSF pulse pressure underlying volume change varied considerably between patients, no correlation between CSF pulse pressure and VPR could be demonstrated.
In order to study more precisely and under controlled conditions the concept of the CSF pulse pressure as a parameter of intracranial volumepressure relationships, a series of experiments was designed in dogs. During rising ICP the relationships of the CSF pulse pressure and the VPR to? the ICP were studied and compared with each other. In another set of experiments the influence on these relationships of variables affecting intracranial haemodynamics, such as blood pressure and arterial $\mathrm{PCO}_{2}$, was investigated. 'These results will be discussed in a subsequent paper.

\section{Methods}

\section{ANALYTICAL METHOD}

The intracranial volume-pressure relationship reflects the elastic properties of the craniospinal system, if the pressure inside the system is compared with the total volume of the system or, since the total volume is not accessible to direct measurement, with additions to this volume. Most authors have approximated the volume-pressure curve by an exponential function (Ryder et al., 1953; Löfgren et al., 1973; Marmarou, 1973). This function was, therefore, chosen as the most simple model to proceed from (Fig. 1):

$$
P=P_{e q} e^{E_{1} V}
$$


where $P=I C P, P_{\text {eq }}=a$ constant equal to the ICP at the equilibrium point (the normal, physiological steady state ICP), $\mathrm{E}_{1}=$ elastance coefficient, and $\mathrm{V}=$ change in total volume of the craniospinal contents with regard to the equilibrium volume $\left(V_{\text {eq }}\right.$. The elastance (E) of the craniospinal system defined as the slope of the volume-pressure curve is the derivative of Equation 1:

$$
\mathrm{E}=\mathrm{dp} / \mathrm{dV}=\mathrm{E}_{1} \mathrm{P}_{\mathrm{eq}} \mathrm{e}^{\mathrm{E}_{1} \mathrm{~V}}=\mathrm{E}_{1} P
$$

So $E_{1}$ determines the elastance at a given pressure and is, therefore, termed the elastance coefficient. $E_{1}$ is the inverse of the pressure-volume index (PVI) defined by Shulman and Marmarou (1971).

When the intracranial volume increases by an amount $\Delta \mathrm{V}$, the intracranial pressure increases by a pressure response $\Delta \mathbf{P}$ :

$$
\mathbf{P}+\Delta \mathbf{P}=\mathbf{P}_{\mathrm{eq}} \mathrm{e}^{\left[\mathrm{E}_{1}(\mathrm{~V}+\Delta \mathrm{V})\right]}
$$

Using equation 1 this equation can be rewritten as:

$$
\Delta \mathbf{P}=\mathbf{P}\left[\mathrm{e}^{\mathrm{E}_{1} \Delta \mathrm{V}}-1\right]
$$

Equation 4 is applicable to the relationships of both the VPR and the CSF pulse pressure to the ICP if $\Delta \mathrm{V}$ is defined as the injected volume during a volume-pressure test and as the transient increase in intracranial blood volume per cardiac cycle respectively. It is the equation of a straight line if $E_{1}$ and $\Delta V$ are constant. The elastance coefficient is constant if the exponential volumepressure model is valid. Since in the case of the volume-pressure test, $\Delta \mathrm{V}$ is constant by definition, a linear correlation between VPR and ICP ought to be accepted as proof of a mono-exponential volume-pressure relationship. The elastance coefficient can be calculated using equation 4:

$$
\mathrm{E}_{1}=\frac{1}{\Delta \mathrm{V}} \ln \left[\frac{\Delta \mathrm{P}}{\mathrm{P}}+1\right]
$$

where $\Delta P / P$ is the slope of the VPR versus ICP relationship.

With regard to the CSF pulse pressure, however, $\Delta \mathrm{V}$ is unknown. Here, the volume change equals the transient increase in CBV per cardiac cycle and is caused by the difference in pulsatile shape of the intracranial arterial inflow and venous outflow of blood. This concept is illustrated in Fig. 2. If CSF pulse pressure and ICP are related linearly it can be inferred that, in case of a constant $E_{1}$, the change in CBV $(\Delta V)$ is also constant. It can be calculated using the following equation, derived from equation 4 :

$$
\Delta V=\frac{1}{E_{1}} \ln \left[\frac{\Delta P}{P}+1\right]
$$

where $\Delta \mathbf{P} / \mathbf{P}$ is the slope of the CSF pulse pressureICP relationship.

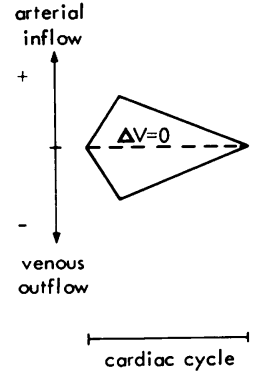

(a)
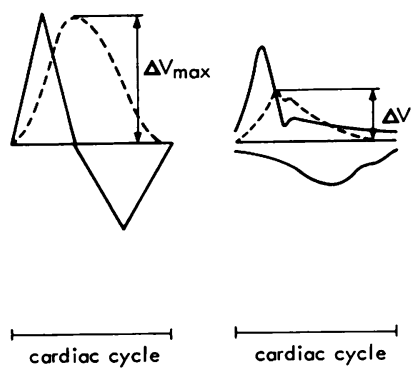

cardiac cycle

(b)
Fig. 2 Diagram illustrating the concept of the transient increase in intracranial blood volume per cardiac cycle $(\Delta V)$. The cerebral arterial inflow and venous outflow curves are schematically drawn; the change in cerebral blood volume is represented by the dashed curve. The magnitude of $\Delta V$ is determined by the interaction between inflow and outflow. If the flow profiles were identical, the resulting volume change would be nil. (a) If inflow and outflow were completely separated, $\Delta V$ would be maximal. (b) Inflow and outflow, however, have different pulsatile flow patterns, thus producing an intermediate volume change (c).

In the experimental study the CSF pulse pressure will be compared with the VPR, so that conclusions can be drawn on $\Delta \mathrm{V}$ and its behaviour with rising ICP.

\section{EXPERIMENTAL METHOD}

Preparation and measurements

The experiments were performed on six adult mongrel dogs of both sexes, weighing from 10 to $19 \mathrm{~kg}$. Anaesthesia was induced with intravenously administered thiopentone $(30 \mathrm{mg} / \mathrm{kg}$ ), and maintained with a mixture of nitrous oxide and oxygen $(2: 1)$ supplemented by chloralose $(50 \mathrm{mg} / \mathrm{kg})$ intravenously. The animals were ventilated using a modified Starling pump, the stroke volume of which was adjusted to maintain normocapnia $\left(\mathrm{PaCO}_{2} 37-43 \mathrm{mmHg}\right)$. Body temperature was maintained between $36^{\circ} \mathrm{C}$ and $38^{\circ} \mathrm{C}$ by heating lamps. Throughout the experiment normal saline was slowly infused intravenously.

The animals were placed in a stereotaxic frame in the sphinx position. The scalp and temporal muscles were reflected. A balloon for cerebral compression, fashioned from glove rubber, was placed epidurally through a burrhole in the right frontotemporal region and connected to a variable speed infusion pump (Sage Instruments, model 351). The ventricular fluid pressure (VFP) was measured bilaterally from needles (inside diameter 
$1.5 \mathrm{~mm}$ ) introduced with the continuous infusion method into the lateral ventricles through small burrholes in the parietal region. All bone defects were sealed with acrylic dental cement. The cisterna magna pressure (CMP) was measured through a needle inserted percutaneously into the cisterna magna. Systemic arterial pressure (SAP) was monitored from an aortic catheter introduced via the femoral artery. Central venous pressure (CVP) was measured in the inferior vena cava through catheterisation of the femoral vein. The electrocardiogram (ECG) was recorded via needle electrodes placed in the limbs of the animal. All pressures were measured continuously by pressure transducers (Bell and Howell) placed at the level of the right atrium. The frequency response of the ventricular needle to manometer system was flat up to $15 \mathrm{~Hz}$ with resonance frequency of $42 \mathrm{~Hz}$ and a damping ratio of 0.25 . The various signals were displayed on two chart recorders (Devices M4 and M2) and stored on a tape recorder (Philips Analog 7). Arterial blood gases were measured at 30 minute intervals throughout the experiment using a direct reading electrode system (Radiometer BMS 3), and values were corrected for temperature differences between the animal and recording system (Severinghaus, 1966).

\section{EXPERIMENTAL PROTOCOL AND DATA COLLECTION}

After all pressures had stabilised the extradural balloon was distended by continuous inflation with normal saline at a rate of $1.0 \mathrm{ml}$ per 15 minutes. As a method of acquiring volume-pressure data the volume-pressure test (VPT) as described by Miller et al. (1973) was used. At intervals of five minutes a cluster of four tests was done by rapid injections of $0.05 \mathrm{ml}$ of normal saline into the left ventricle using a three-way stopcock interposed in the tubing connecting the ventricular needle to the pressure transducer. The VPR was read from the injected ventricle and calculated from the immediate change in the mean VFP in corresponding parts of the VFP respiratory cycle. All mean pressures including SAP were calculated from the diastolic pressure plus one-third of the pulse pressure. In order to reduce variability from errors inherent in the manoeuvre, the mean VPR of each cluster of four tests was taken and related to the mean preinjection VFP (mean baseline VFP). After completion of the four tests the VFP always returned to the steady state level before the next time interval was reached.

During the volume-pressure tests the speed of the chart recorder was increased, so that the amplitude of the CSF pulse could be distinguished clearly. Eight pulse pressures were calculated by taking the average value of the pulse pressures over the last respiratory cycle before and the first cycle after each VPT. The means of the four pulse pressures before and after each test were related to the mean baseline and peak VFP respectively. As a result of this procedure each time interval yielded one VPR and two pulse pressures.

When changes in SAP occurred, as they sometimes did in the advanced stages of cerebral compression, no special measures were taken to keep the SAP at a constant level. However, since changes in SAP are known to affect both the intracranial volume-pressure relationship (Löfgren, 1973; Leech and Miller, 1974b) and the CSF pulse pressure, all values of both VPR and pulse pressure obtained at levels of SAP deviating more than $15 \%$ from the mean SAP were discarded.

The experiment was terminated when the VFP had reached the level of the blood pressure, except for three animals in which it was continued far beyond this level. The brains were transected at the foramen magnum (brain weights between 720 and $92 \mathrm{~g}$ ), fixed in formalin and sectioned coronally at $5 \mathrm{~mm}$ intervals for pathology. Proper placement of the ventricular needles was verified and major brain lesions from surgical preparation were excluded.

\section{Results}

RELATIONSHIP BETWEEN VOLUME-PRESSURE RESPONSE AND VENTRICULAR FLUID PRESSURE In the plots of VPR versus VFP (Fig. 3) three more or less clearly definable zones could be observed. At first the VPR increased linearly with the VFP. Next the VPR levelled off or even decreased, and finally at the advanced stage of cerebral compression the response started to rise again, but more rapidly than before. The regression equations of the first zone are given in Table 2. These linear relationships are in accordance with the mono-exponential volume-pressure model, implying a constant elastance coefficient $\left(E_{1}\right) . E_{1}$ was calculated from the slopes of the regression lines using equation 5 and the results are given in Table 2 . The data with regard to the breakpoint between first and second zone are given in Table 3 . It occurred at a mean VFP of $60 \mathrm{mmHg}$, but with a considerable variation between the animals. There was a positive linear correlation between VFP and SAP at the breakpoint $(r=0.82, \mathrm{P}<0.05)$, and consequently the 

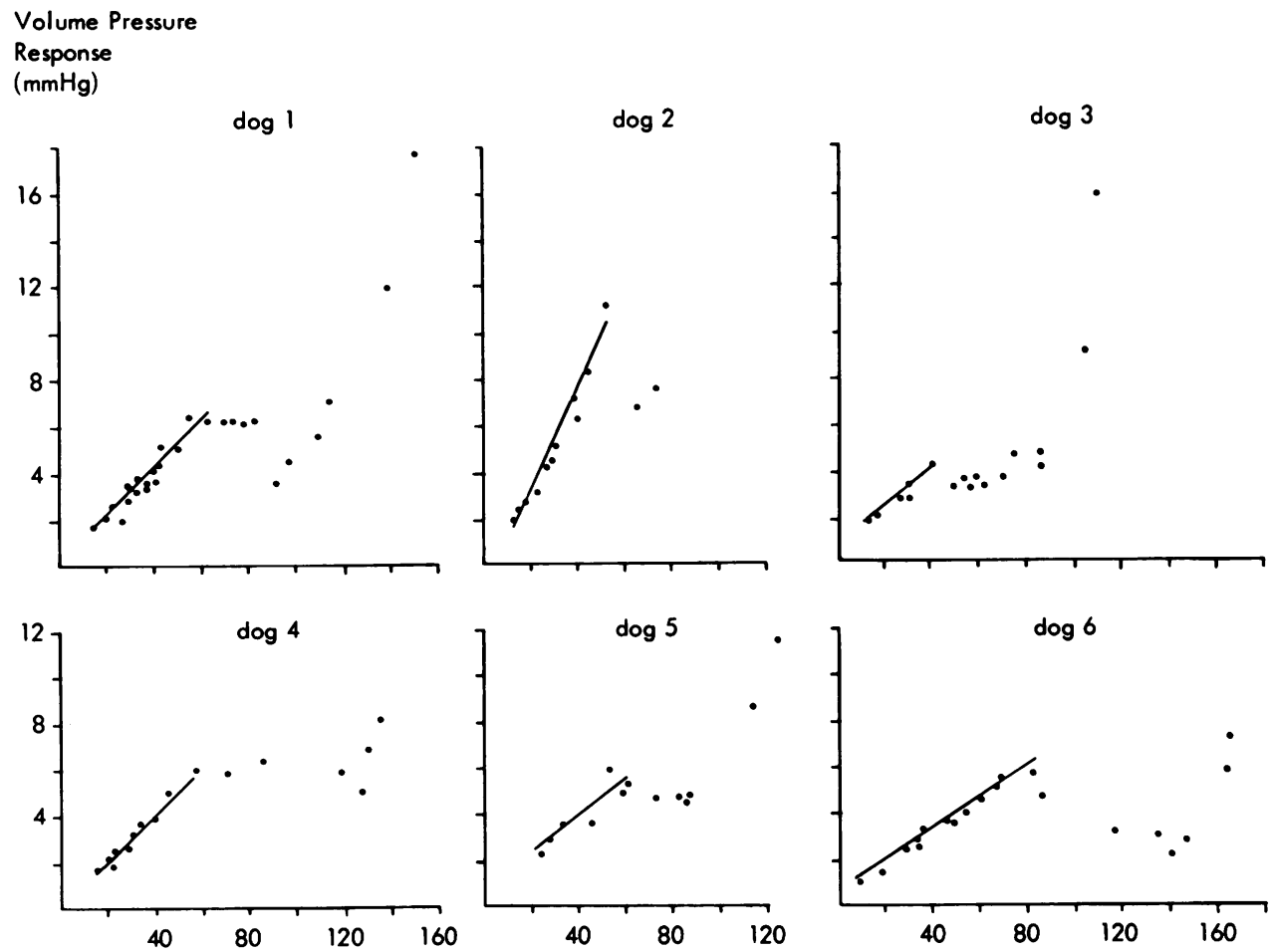

Ventricular Fluid Pressure $(\mathrm{mmHg})$

Fig. 3 Plots of the volume-pressure response (VPR) against the ventricular fluid pressure $(V F P)$ during continuous inflation of an extradural balloon in six animals. Three different zones can be recognised. In the first zone the VPR increases linearly with the VFP.The regression lines are drawn and the equations are given in Table 2 . In the second zone the $V P R$ remains constant and!or decreases, and finally the VPR increases again but more rapidly than in the first zone.

Table 2 Relationship between volume-pressure response (VPR) and ventricular fluid pressure (VFP): regression equations and elastance coefficients $\left(E_{1}\right)$ below and mean VPR $( \pm S D)$ and elastance above the first breakpoint

\begin{tabular}{|c|c|c|c|c|c|c|c|c|c|}
\hline \multirow[t]{2}{*}{ Animal } & \multicolumn{5}{|c|}{ Below first breakpoint } & \multicolumn{4}{|c|}{ Above first breakpoint } \\
\hline & $\begin{array}{l}\text { Regression } \\
\text { equations }\end{array}$ & $n$ & $r$ & $\begin{array}{l}E_{1} \\
(1 / m l)\end{array}$ & $\begin{array}{l}\text { VFP range } \\
(\mathrm{mm} \mathrm{Hg})\end{array}$ & $\begin{array}{l}\text { Mean VPR } \\
(m m H g)\end{array}$ & $n$ & $\begin{array}{l}\text { Elastance } \\
(m m H g / m l)\end{array}$ & $\begin{array}{l}\text { VFP range } \\
(m m H g)\end{array}$ \\
\hline $\begin{array}{l}1 \\
2 \\
3 \\
4 \\
5 \\
6\end{array}$ & $\begin{array}{l}y=0.10 x-0.1 \\
y=0.22 x-1.5 \\
y=0.08 x+0.7 \\
y=0.10 x+0.0 \\
y=0.08 x+0.8 \\
y=0.07 x+0.6\end{array}$ & $\begin{array}{r}18 \\
11 \\
6 \\
10 \\
7 \\
13\end{array}$ & $\begin{array}{l}0.95 \\
0.98 \\
0.97 \\
0.99 \\
0.89 \\
0.99\end{array}$ & $\begin{array}{l}1.9 \\
4.0 \\
1.5 \\
2.0 \\
1.5 \\
1.3\end{array}$ & $\begin{array}{r}17.1-66.3 \\
13.4-52.0 \\
14.3-41.6 \\
14.8-57.7 \\
24.0-60.1 \\
8.8-81.0\end{array}$ & $\begin{array}{l}5.5 \pm 1.0 \\
7.1 \pm 0.6 \\
3.7 \pm 0.6 \\
5.7 \pm 0.6 \\
4.7 \pm 0.2 \\
3.2 \pm 0.9\end{array}$ & $\begin{array}{l}8 \\
2 \\
9 \\
5 \\
5 \\
5\end{array}$ & $\begin{array}{r}110 \\
142 \\
74 \\
114 \\
94 \\
64\end{array}$ & $\begin{array}{l}66.3-111.8 \\
65.4-72.5 \\
49.4-87.2 \\
57.7-126.5 \\
60.1-85.0 \\
85.3-145.5\end{array}$ \\
\hline Mean & $y=0.11 x+0.1$ & & & 2.0 & $15.4-59.8$ & $5.0 \pm 1.4$ & & 100 & $63.9-104.8$ \\
\hline
\end{tabular}

variation was much narrower if the cerebral perfusion pressures (CPP) were considered. No relation could be demonstrated between the breakpoint and transtentorial herniation (Table 4). Early herniation was defined as the appearance of a pressure gradient between VFP and CMP. When
CMP did not rise any further and no VPR could be detected in the CMP tracing, tentorial obstruction was considered complete.

The second zone in the plot of VPR versus VFP poses a problem with regard to the analysis of the data. Less data points were available, as the 
Table 3 Ventricular fluid pressure $(V F P)$, systemic arterial pressure $(S A P)$, cerebral perfusion pressure $(C P P)$, and balloon volume at first and second breakpoints

\begin{tabular}{|c|c|c|c|c|c|c|c|c|}
\hline \multirow[t]{2}{*}{ Animal } & \multicolumn{4}{|c|}{ First breakpoint } & \multicolumn{4}{|c|}{ Second breakpoint } \\
\hline & $\begin{array}{l}V F P \\
(m m H g)\end{array}$ & $\begin{array}{l}S A P \\
(m m H g)\end{array}$ & $\begin{array}{l}C P P \\
(m m H g)\end{array}$ & $\begin{array}{l}\text { Balloon } \\
\text { volume }(\mathrm{ml})\end{array}$ & $\begin{array}{l}V F P \\
(m m H g)\end{array}$ & $\begin{array}{l}S A P \\
(m m H g)\end{array}$ & $\begin{array}{l}C P P \\
(m m H g)\end{array}$ & $\begin{array}{l}\text { Balloon } \\
\text { volume }(\mathrm{ml})\end{array}$ \\
\hline $\begin{array}{l}1 \\
2 \\
3 \\
4 \\
5 \\
5\end{array}$ & $\begin{array}{l}66.3 \\
52.0 \\
41.6 \\
57.7 \\
60.1 \\
81.0\end{array}$ & $\begin{array}{l}162.3 \\
128.3 \\
126.6 \\
156.0 \\
134.3 \\
164.0\end{array}$ & $\begin{array}{l}96.0 \\
76.3 \\
85.0 \\
98.3 \\
74.2 \\
83.0\end{array}$ & $\begin{array}{l}5.7 \\
3.3 \\
1.7 \\
3.0 \\
2.0 \\
4.0\end{array}$ & $\begin{array}{l}111.8 \\
104.6 \\
130.3 \\
112.5 \\
163.9\end{array}$ & $\begin{array}{l}136.7 \\
116.0 \\
153.7 \\
131.3 \\
165.7\end{array}$ & \begin{tabular}{r|}
24.9 \\
11.4 \\
23.4 \\
18.8 \\
1.8
\end{tabular} & $\begin{array}{l}8.3 \\
6.0 \\
5.0 \\
5.3 \\
6.7\end{array}$ \\
\hline Mean & 59.8 & 145.3 & 85.5 & 3.3 & 124.6 & 140.7 & 16.1 & 6.3 \\
\hline
\end{tabular}

Table 4 Ventricular fluid pressure (VFP) and balloon volume at early and complete tentorial obstruction

\begin{tabular}{llllll}
\hline Animal & $\begin{array}{l}\text { Early tentorial } \\
\text { obstruction }\end{array}$ & $\begin{array}{l}\text { Complete tentorial } \\
\text { NFP }\end{array}$ & $\begin{array}{l}\text { Balloon } \\
\text { obstruction }\end{array}$ & \\
\cline { 2 - 3 } \cline { 5 - 6 } & $(\mathrm{mmHg})$ & volume $(\mathrm{ml})$ & & $\begin{array}{l}\text { VFP } \\
(\mathrm{mmHg})\end{array}$ & $\begin{array}{l}\text { Balloon } \\
\text { volume }(\mathrm{ml})\end{array}$ \\
\hline 1 & 52.3 & 5.3 & 72.7 & 6.7 \\
2 & 16.3 & 0.7 & 24.7 & 1.7 \\
3 & 24.5 & 1.0 & 54.3 & 2.7 \\
4 & 35.0 & 2.3 & 114.0 & 4.0 \\
5 & 48.5 & 1.3 & 79.0 & 2.7 \\
6 & 48.3 & 2.7 & 113.0 & 4.7 \\
\hline Mean & 37.5 & 2.2 & 76.3 & 3.8 \\
\hline
\end{tabular}

animals passed more rapidly through this phase of the experiment and the blood pressure had sometimes fallen below acceptable levels. In addition, the VPR in the injected ventricle was often small in contrast with a much higher response in the other ventricle, the latter consisting of a high peak with a very rapid return to the preinjection level (Fig. 4). In those cases there was never reason to doubt the patency of the ventricular needle. The high peak response is apparently missed in the injected ventricle because of the time factor involved in turning the three-way stopcock in the recording position. The rapid return of the pressure to the baseline suggests a very rapid compensation of the volume added. In view of these findings we believe that the fall in VPR, at least in part, is an artefact produced by failure of the volume-pressure test to detect rapid spatial compensatory mechanisms. Figure 5 shows the VPR in both ventricles in one of the experimental animals plotted against the VFP, demonstrating that the phenomenon described is responsible for the decrease in VPR, but does not explain the levelling off. Unfortunately, these observations could not be made in all animals with falling VPRs as the recording needle in the non-injected ventricle was sometimes blocked a this stage. This VFP range was, therefore, con 2 ceived as a zone with a constant VPR defined bo the mean VPR (Table 2). The implication is thag the volume-pressure relationship is no longe exponential but linear in nature (constants. elastance), and consequently the mathematica? model cannot be applied. The elastance was calculated by using $\mathrm{E}=\Delta \mathrm{P} / \Delta \mathrm{V}=\mathrm{VPR} / 0.05 \mathrm{mmHg} /$ $\mathrm{ml}$ (Table 2).

The data with regard to the second breakpoint in the VPR-VFP relationship at which the VPR began to increase again are given in Table 3. This occurred at a mean VFP of $125 \mathrm{mmHg}$. In each

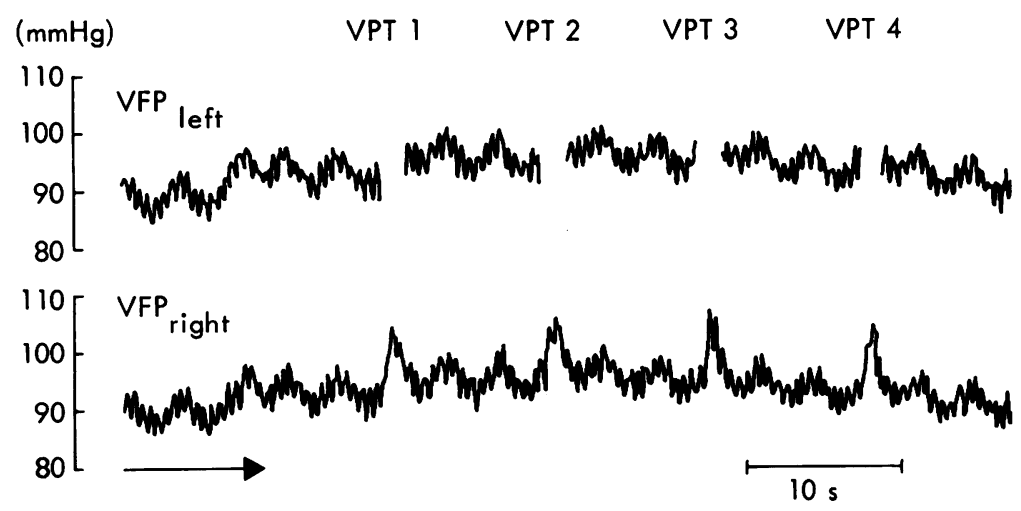

Fig. 4 Comparison of the volume-pressure response (VPR) in the injected (upper tracing) and the non-injected ventricle (lower tracing) during four volume-pressure tests (VPT) performed at high ventricular fluid pressures $(V F P)$ in a single animal. The high pressure spike observed in the non-injected ventricle is missed in the other ventricle because of the time factor involved in turning the three-way stopcock into the recording position. 
VPR
$(\mathrm{mm} \mathrm{Hg})$

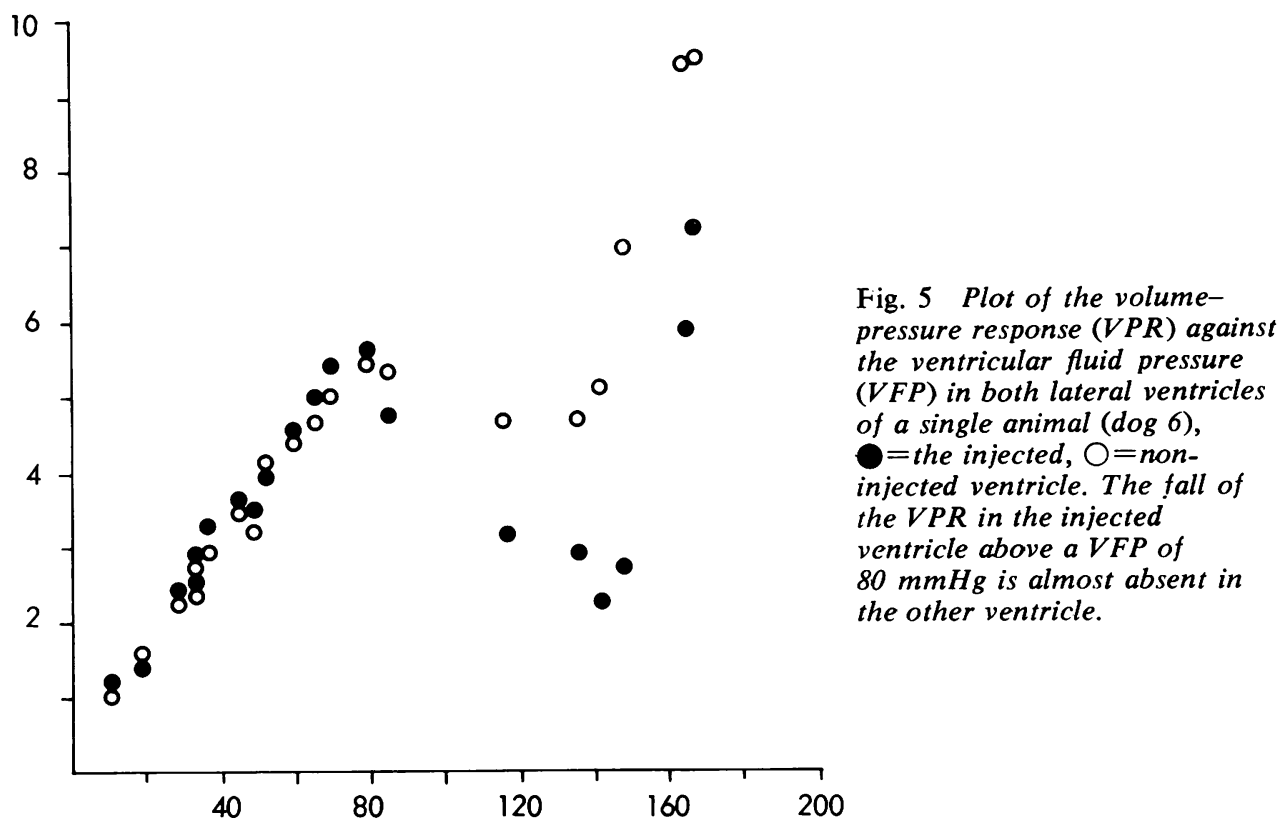

$\operatorname{VFP}(\mathrm{mm} \mathrm{Hg})$

animal the breakpoint was related to the level of the diastolic blood pressure. In three animals sufficient data were available to calculate the regression lines for the pressure range above this break point (Table 5). The slopes were always steeper than in the first pressure zone.

\section{RELATIONSHIP BETWEEN CSF PULSE PRESSURE AND} VENTRICULAR FLUID PRESSURE

The relationship between CSF pulse pressure and VFP is demonstrated in Fig. 6. The pulse pressure increased linearly with rising intracranial pressure but more rapidly in the high pressure range although this was not equally distinct in each animal. When the VFP approached the level of the blood pressure the amplitude of the CSF pulse levelled off, and finally decreased above this level.

Table 5 Regression equations of the relationship between volume-pressure response and ventricular fluid pressure (VFP) above the level of the diastolic blood pressure

\begin{tabular}{lllll}
\hline Animal & $\begin{array}{l}\text { Regression } \\
\text { equations }\end{array}$ & $n$ & $r$ & $\begin{array}{l}\text { VFP range } \\
(\mathrm{mmHg})\end{array}$ \\
\hline 1 & $\mathrm{y}=0.26 \mathrm{x}-23.6$ & 4 & 0.98 & $111.8-155.3$ \\
3 & $\mathrm{y}=0.27 \mathrm{x}-15.4$ & 5 & 0.93 & $104.6-159.2$ \\
6 & $\mathrm{y}=0.31 \mathrm{x}-44.7$ & 4 & 1.00 & $163.9-207.8$ \\
\hline
\end{tabular}

Since the VPR versus VFP plots had shown three pressure zones with different volume-pressure relationships, we decided to analyse the CSF pulse pressure data for each zone separately. The regression lines are given in Table 6 . In all but one animal there was a significant difference in slope on either side of the breakpoint, the slope always becoming steeper ( $\mathrm{F}$ distribution, $\mathrm{P}<0.01$ in four animals and $P<0.05$ in one). Variables with possible effect on the CSF pulse pressure, such as systemic arterial pressure, arterial pulse pressure, and heart rate, remained stable throughout the experiment (Table 7).

The linearity of the relationship between CSF pulse pressure and VFP has a different meaning on either side of the breakpoint if the volumepressure relationship is taken into consideration. As an example, Fig. 7 shows a comparison of the two relationships in one of the animals. Below the breakpoint, where the concept of a monoexponential volume-pressure curve holds true (constant $E_{1}$ ), it means according to equation 4 , that the change in CBV per cardiac cycle $(\Delta \mathrm{V})$ is also constant. Using equation $6, \Delta \mathrm{V}$ was calculated for the various animals (Table 6): $\Delta \mathrm{V}$ varied considerably, which explains the lack of correspondence between the slopes of the CSF pulse pressure versus VFP regression lines and the 
Fig. 6 Plots of the CSF pulse pressure against the ventricular fluid pressure (VFP) during continuous inflation of an extradural balloon in six animals. The pulse pressure increases linearly with the VFP up to the level of the blood pressure but with a breakpoint. The regression lines are drawn and the equations are given in Table 6. The slope of the regression line above the breakpoint was always steeper except for animal 3.

Table 6 Relationship between CSF pulse pressure and ventricular fluid pressure: regression equations, volume change per cardiac cycle $(\Delta V)$ below and increase in $\Delta V$ above the first breakpoint

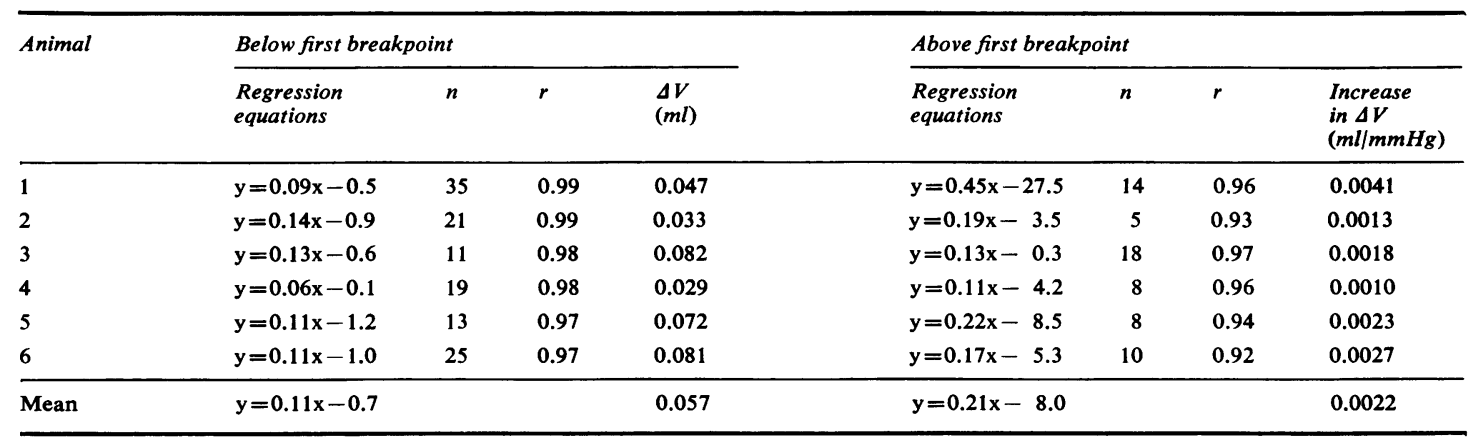


Table 7 Systemic arterial pressure (SAP), arterial pulse $(\triangle S A P)$, and heart rate $(H R)$ below and above the first breakpoint $(M \pm S D)$

\begin{tabular}{|c|c|c|c|c|c|c|}
\hline \multirow[t]{2}{*}{ Animal } & \multicolumn{3}{|c|}{ Below first breakpoint } & \multicolumn{3}{|c|}{ Above first breakpoint } \\
\hline & $\begin{array}{l}S A P_{m} \\
(\boldsymbol{m m H g})\end{array}$ & $\begin{array}{l}\Delta S A P_{m} \\
(m m H g)\end{array}$ & $\begin{array}{l}H R \\
\text { (beats/min) }\end{array}$ & $\begin{array}{l}S A P_{m} \\
(\boldsymbol{m} \boldsymbol{m} \boldsymbol{H} g)\end{array}$ & $\begin{array}{l}\Delta S A P_{m} \\
(m m H g)\end{array}$ & $\begin{array}{l}\text { HR } \\
\text { (beats/min) }\end{array}$ \\
\hline 1 & $159.5 \pm 8.1$ & $56.3 \pm 8.3$ & $114.2 \pm 11.4$ & $144.9 \pm 11.9$ & $54.1 \pm 6.4$ & $114.0 \pm 12.4$ \\
\hline 2 & $126.4 \pm 2.6$ & $39.8 \pm 1.3$ & $151.5 \pm 10.7$ & $122.0 \pm 2.8$ & $39.5 \pm 4.9$ & $134.5 \pm 3.5$ \\
\hline 3 & $118.8 \pm 5.8$ & $34.0 \pm 1.7$ & $132.0 \pm 3.3$ & $119.6 \pm 4.7$ & $35.1 \pm 1.5$ & $134.7 \pm 2.8$ \\
\hline 4 & $151.6 \pm 3.6$ & $46.1 \pm 2.2$ & $144.5 \pm 5.3$ & $156.4 \pm 6.2$ & $43.7 \pm 2.6$ & $175.0 \pm 14.2$ \\
\hline 5 & $135.6 \pm 2.9$ & $53.9 \pm 6.6$ & $165.3 \pm 1.6$ & $130.8 \pm 9.3$ & $50.3 \pm 2.1$ & $168.3 \pm 2.3$ \\
\hline 6 & $167.8 \pm 1.8$ & $50.8 \pm 4.3$ & $168.0 \pm 6.6$ & $158.4 \pm 4.7$ & $46.0 \pm 4.2$ & $156.0 \pm 9.5$ \\
\hline Mean & $143.3 \pm 19.4$ & $46.8 \pm 8.6$ & $145.9 \pm 20.5$ & $138.7 \pm 17.0$ & $44.6 \pm 7.0$ & $147.1 \pm 23.3$ \\
\hline
\end{tabular}

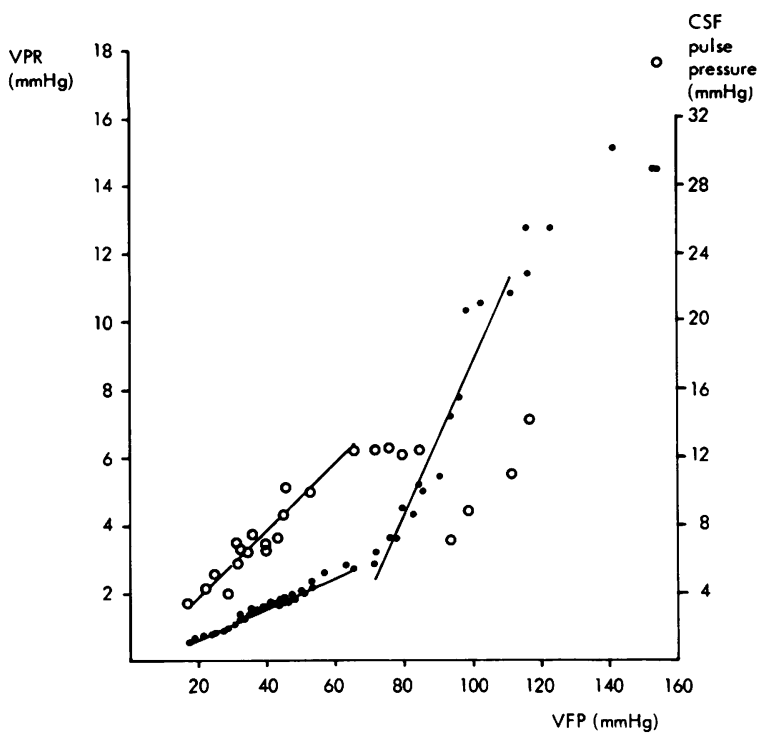

Fig. 7 Plot of the volume-pressure response $(O)$ and the CSF pulse pressure (O) against the ventricular fluid pressure (VFP) in a single animal $(\operatorname{dog} 1)$.

magnitude of the elastance coefficients. The variation in $\Delta V$ could not be explained from differences in SAP, arterial pulse pressure and heart rate.

Above the breakpoint, where a linear volumepressure relationship exists, the increase in CSF pulse pressure is only related to the change in CBV per cardiac cycle according to: $\Delta \mathrm{P}=\mathrm{E} \Delta \mathrm{V}$, where $\mathrm{E}$ (elastance) is constant. Consequently, in this pressure zone $\Delta \mathrm{V}$ is increasing with rising intracranial pressure. The increase in $\Delta \mathrm{V}$ per $\mathrm{mmHg}$ was calculated and the results are given in Table 6.

In the animals with data above the level of the SAP no specific analysis was possible. A decrease in CSF pulse pressure was generally observed.

\section{Discussion}

The present series of experiments was designed to study the relation of the CSF pulse pressure to both ICP and the VPR with the clinically motivated objective of investigating whether the ratio of pulse pressure to ICP could serve as a reliable guide in the assessment of the craniospinal volume-pressure relationship. The results, however, also give rise to a critical review of the volume-pressure function itself.

\section{VOLUME-PRESSURE RELATIONSHIPS}

The validity of the mono-exponential volumepressure model follows directly from the linear correlation of VPR with VFP. This is consistent 
Ventricular fluid pressure CSF pulse pressure ( $\mathrm{mm} \mathrm{Hg}$ )

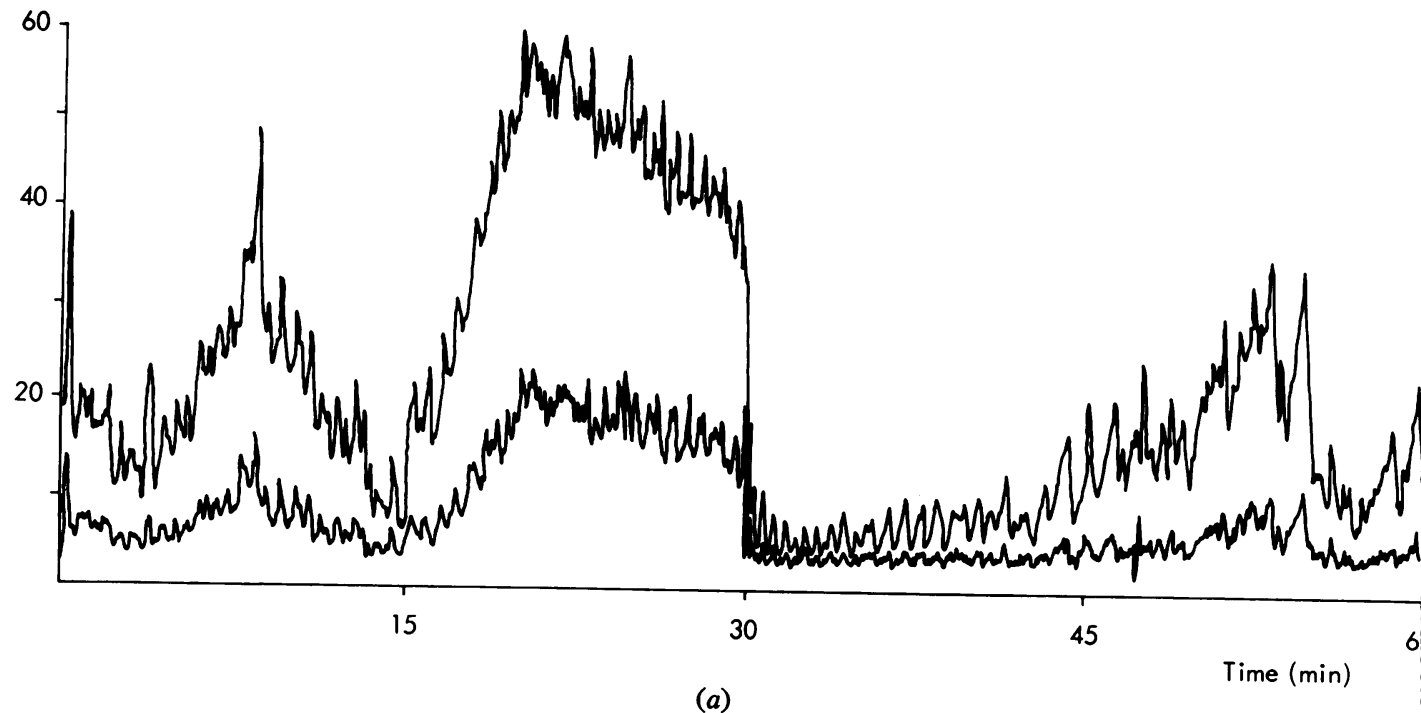

CSF

pulse

pressure

( $\mathrm{mm} \mathrm{Hg}$ )
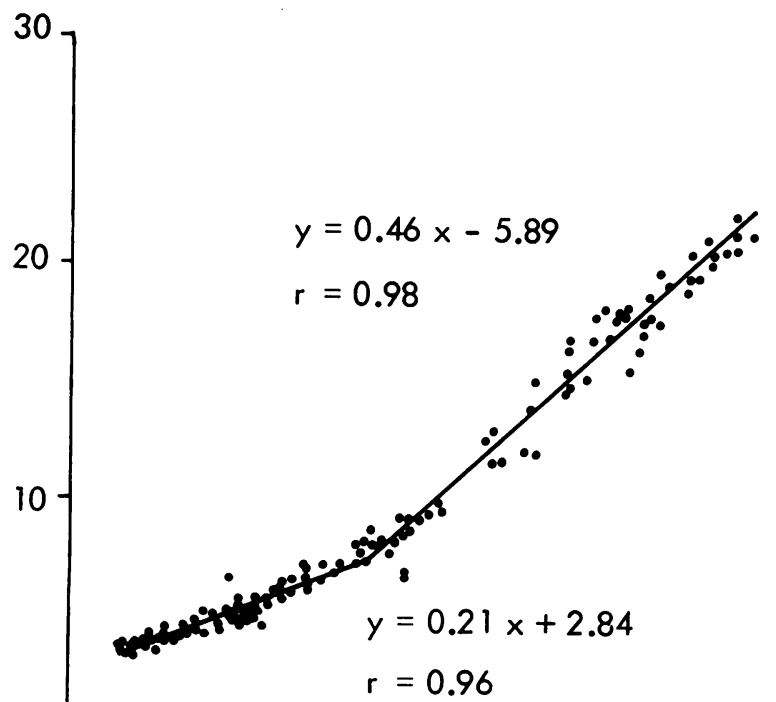

Fig. 8 (a) Computer plot of the ventricular fluid pressure (VFP, upper tracing) and the CSF pulse pressure (lower tracing) during $V F P$ monitoring in a patient showing a classical plateau wave and two "abortive" waves. The method of computer analysis has been reported previously (Avezaat et al., 1976). (b) Computer plot of the relationship between the CSF pulse pressure and the VFP of the pressure recording shown in (a). A breakpoint occurs at a $V F P$ of about $25 \mathrm{mmHg}$ demonstrating a steeper linear relationship during the plateau wave.
20

(b)

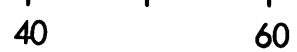

$\operatorname{VFP}(\mathrm{mm} \mathrm{Hg})$ 
with the findings of other authors (Ryder et al., 1953; Löfgren et al., 1973; Marmarou, 1973; Miller et al., 1973). The volume-pressure parameter used in this study, $\mathrm{E}_{1}$, determines the slope of the volume-pressure curve or the elastance at a given ICP and was, therefore, termed the "elastance coefficient." A larger $\mathrm{E}_{1}$ means a steeper slope. Sullivan and his colleagues (1977), using a similar mathematical model, have recently reported a progressive elevation of the PVI (the inverse parameter of $E_{1}$ ) during cerebral compression in cats. This invalidates the monoexponential model which implies a constant PVI. However, when their elastance data are plotted against the ICP, instead of balloon volume, a linear relationship is found confirming the monoexponential model. This contradiction is caused by the mathematical model used, which implies that the linear relationship of elastance (VPR) versus ICP passes through the origin of the axes. As long as the regression lines have their intercepts close to the origin, as in this study, the model will not be seriously disturbed. If not, the mathematical model must be extended by a constant term as we have previously reported (Avezaat et al., 1976). When this extended model is applied to the data of Sullivan et al. (1977) the PVI remains constant up to an ICP of about $60 \mathrm{mmHg}$; so the mono-exponential volumepressure model holds true. The constant term has the effect of shifting the volume-pressure curve along the pressure axis, which was demonstrated by Löfgren $e t$ al. (1973) to be related to the central venous pressure.

Confusion has been caused in the past when, in discussing volume-pressure relationships, reference was made to curves constructed by plotting the steady state ICP against the volume of an expanding intracranial mass (Langfitt et al., 1965; Leech and Miller, 1974a). However clinically relevant they may be, these curves do not reflect the elastic properties of the system as they involve the process of spatial compensation. This is in agreement with the results of the present study which failed to show a correspondence between $E_{1}$ and the balloon volume accommodated over the ICP range for which $E_{1}$ was valid. For example, animal 2 has a relatively large spatial compensatory capacity and a steep volume-pressure curve. The large $\mathrm{E}_{1}$ of this animal may be explained by the early occurrence of tentorial herniation. Other investigators ( $\mathrm{Lim}$ et al., 1973; Löfgren and Zwetnow, 1973; Marmarou et al., 1975) have reported an increase of the elastance when the communication between the cranial and spinal compartment was impaired. In this series herniation always started to develop before the breakpoint, which may account for the generally large $\mathrm{E}_{1}$ compared to results obtained with rapid infusion techniques.

An important finding in this study was the validity of the exponential volume-pressure model for a restricted ICP range only. At a mean ICP of $60 \mathrm{mmHg}$ a breakpoint occurred above which the volume-pressure relationship became a linear function with a slope equal to the elastance at that point. In order to understand this the question should be raised as to why the relationship is exponential in the first place. The magnitude of the change in ICP in response to a rapid volume addition is determined by two factors constituting the so-called elastic properties of the craniospinal system. The first factor is related to the physical properties of the craniospinal contents and their confining structures. Here, the "give" in the system depends largely on the distensibility of the spinal dural sac (Martins et al., 1972). The characteristics of these structures, like those of all other soft body tissues, are essentially nonlinear (King and Lawton, 1950). The second factor is related to disturbances in the intracranial haemodynamics. The volume added compresses the venous outflow section and, through an increase of the outflow resistance, produces a rise in the upstream intravascular pressure, which in turn is transmitted to the surrounding CSF. The pressure response is thus determined by the compressibility of the venous vascular bed. According to Poiseuille's law the resulting venous pressure is inversely proportional to the square of vascular volume, which provides further support for the nonlinear nature of the volume-pressure relationship. From Poiseuille's law also follows that the same volume increment will result in a lower pressure response, if flow is reduced. So progressive reduction of cerebral blood flow (CBF) contributes to the linear shape of the volume-pressure curve above the breakpoint, and the breakpoint may consequently be related to failure of autoregulation. Löfgren et al. (1973) described the volumepressure curve from an ICP of $20 \mathrm{mmHg}$ up to the level of the blood pressure as a linear function (high pressure elastance). The curve was established during rapid infusion in the cisterna magna of dogs within a time interval too short for an autoregulatory vasomotor response to develop. Their results are, therefore, in agreement with the concept that a linear volume-pressure relationship is related to a decrease in CBF. Furthermore, the ICP at which Johnston et al. (1973), in a 
similar preparation in the baboon, found a reduction of CBF corresponds with the pressure at the breakpoint of this study: $60 \mathrm{mmHg}$. The narrower range of CPP than of ICP at the breakpoints is another argument for relating this point to changes in intracranial haemodynamics.

From the difference in VPR between injected and non-injected ventricles we conclude that the decrease of the elastance close to the level of the blood pressure, an observation also made by others (Löfgren et al., 1973; Leech and Miller, 1974a), is an artefact. This artefact is caused by a rapid spatial compensatory mechanism, probably displacement of venous blood. So the VPR at this level of ICP is no longer an accurate measure of the elastance. Above the blood pressure level, where CBF has ceased completely, the cranial compartment fulfils the requirements of the Monro-Kellie doctrine resulting in a very steep volume-pressure relationship.

\section{CSF PULSE PRESSURE}

The linear increase of the CSF pulse pressure with rising ICP is consistent with observations made in patients (Janny, 1974; Avezaat et al., 1976; Nornes et al., 1977; Szewczykowski et al., 1977). In this study we found a breakpoint above which the pulse amplitude increased more rapidly and which coincided with a breakpoint in the volumepressure relationship. From the comparison between pulse pressure and VPR we concluded that the change in CBV per cardiac cycle $(\Delta V)$ remains constant up to the level of the ICP at the breakpoint. The important implication is that in this ICP range changes in the slope of the CSF pulse pressure versus ICP relationship truly reflect changes in the volume-pressure relationship. Above the breakpoint the increase in pulse pressure is, on account of the constant elastance, caused by a progressive increase of $\Delta \mathrm{V}$.

Because of the coincidence of the two breakpoints it is plausible that the increase of $\Delta \mathrm{V}$, like the levelling off of the VPR, is caused by haemodynamic changes, particularly failure of autoregulation and reduction of CBF. Symon et al. (1974, 1975), using the pulse transmission from pial artery to Labbé's vein as an index of cerebrovascular resistance (CVR), found a linear increase of the pulse transmission up to an ICP of $70 \mathrm{mmHg}$. From this point onwards autoregulation began to fail, $\mathrm{CBF}$ decreased, and the pulse transmission increased more steeply. We (Avezaat et al., 1976) have reported an increased ratio of CSF pulse pressure to ICP during plateau waves (Lundberg, 1960). The cause of plateau waves has been attributed to cerebral vasodilatation and increased CBV (Lundberg et al., 1968).

A physiological explanation for the increase of $\Delta \mathrm{V}$ can be offered using the concept on the origin of the change in CBV during a cardiac cycle (Fig. 2). The magnitude of this volume change is determined by the difference in shape between the pulsatile arterial inflow and venous outflow curves. These flow profiles depend on the impedances of the arterial and venous sections of the cerebral vasculature. The resistance vessels dilate with rising ICP as part of the autoregulatory process, causing a decrease of the inflow impedance and a more rapid inflow of blood. The outflow impedance at the same time increases as a result of venous compression, thus delaying the outflow of blood. As a result, inflow and outflow gradually become two more or less separate processes (Fig. 2b). This implies an increase in $\Delta \mathrm{V}$ as soon as ICP starts to rise, which, according to the analytical model, corresponds with a nonlinear relationship between pulse pressure and ICP. Evidence for this theory can be derived from the work of Guinane (1975) who described this relationship by an exponential function. In the ICP range below the breakpoint the changes in inflow and outflow impedance are probably relativelyo small, so that a linear relationship implying a constant $\Delta \mathrm{V}$ is a good approximation. When autoregulation fails and progressive vasoparalysise occurs (Langfitt et al., 1965) the inflow impedance is at its lowest. At the same time the resistance at the outflow tract continues to increase, to which compression of the dural sinuses might be a contributory factor (Shapiro et al., 1966). This explains the marked increase in $\Delta \mathrm{V}$ above the breakpoint. The paradox of an increase in $\Delta \mathrm{V}$ in spite of a reduction in CBF can be solved by looking more closely at the cerebral inflow section. A decrease in CBF does not necessarily mean a decrease in the arterial inflow of blood but simply an increase in arterial reflux. Nornes et al. (1977), measuring electromagnetic flow in the internal carotid artery of patients, found a reduction of flow at high levels of ICP but at the same time an increase of pulsatile flow. This increase was most marked at a breakpoint where also the ICP pulse increased more rapidly.

Another observation was the occurrence of reverse flow during diastole. This also explains why large pulse pressures can still be recorded when the ICP has been raised above the level of the blood pressure and consequently complete circulatory arrest exists. In this case both inflow and outflow take place at the arterial side. 


\section{CLINICAL IMPLICATIONS}

The volume-pressure test is generally recognised as the most practical method of assessing the volume-pressure relationship in patients. The disadvantages of the test have been mentioned. Moreover, the test gives only a narrow view of the volume-pressure curve. The heart, on the other hand, is generating volume-pressure tests continuously. The relationship between the CSF pulse pressure and the ICP can be processed at the bedside by simple electronic equipment on the basis of which the volume-pressure function can be assessed continuously. However, since the change in CBV per cardiac cycle varies between patients as between the animals of this study, a VPT is still needed at the beginning of an ICP recording to determine the magnitude of $\Delta \mathrm{V}$. In the course of ICP monitoring $\Delta \mathrm{V}$ may be affected by factors changing cerebral haemodynamics, such as arterial carbon dioxide tension, blood pressure, and arterial pulse pressure. The influence of these variables on $\Delta \mathrm{V}$ will be dealt with in a subsequent study.

An important finding of this study was that a marked increase in the CSF pulse pressure to ICP ratio may indicate loss of autoregulation. In that case a relatively low VPR can be expected as a result of the levelling off phenomenon and the rapid spatial compensatory mechanism. Consequently, in non-autoregulating patients the CSF pulse pressure is a better parameter of the clinical state than the VPR.

This study was supported by a grant from the Netherlands Organisation for the Advancement of Pure Research (ZWO). We would like to express our appreciation to the staffs of the Institute of Neurological Sciences and the Wellcome Surgical Research Institute, Glasgow for their assistance.

\section{References}

Avezaat, C. J. J., van Eijndhoven, J. H. M., de Jong, D. A., and Moolenaar, W. C. J. (1976). A new method of monitoring intracranial volume-pressure relationships. In Intracranial Pressure III, pp. 308313. Edited by J. W. F. Beks, D. A. Bosch, and M. Brock. Springer: Berlin.

Castel, J. P., and Cohadon, F. (1976). The pattern of cerebral pulse: automatic analysis. In Intracranial Pressure III, pp. 303-307. Edited by J. F. W. Beks, D. A. Bosch, and M. Brock. Springer: Berlin.

Guinane, J. E. (1975). Cerebrospinal fluid pulse pressure and brain compliance in adult cats. Neurology (Minneapolis), 25, 559-564.

Janny, P. (1974). La surveillance de la pression intracranienne en neuro-chirurgie. Aspects graphiques et séméiologiques de la pression intra-cranienne. Neurochirurgie, 20, 521-534.
Johnston, I. H., Rowan, J. O., Harper, A. M., and Jennett, W. B. (1973). Raised intracranial pressure and cerebral blood flow. 2. Supratentorial and infratentorial mass lesions in primates. Journal of Neurology, Neurosurgery, and Psychiatry, 36, 161170.

King, A. L., and Lawton, R. W. (1950). The elasticity of soft body tissues. Scientific Monthly, 71, 258-260.

Langfitt, T. W., Weinstein, J. D., and Kassell, N. F. (1965). Cerebral vasomotor paralysis produced by intracranial hypertension. Neurology (Minneapolis), 15, 622-641.

Leech, P., and Miller, J. D. (1974a). Intracranial volume-pressure relationships during experimental brain compression in primates. 1. Pressure responses to changes in ventricular volume. Journal of Neurology, Neurosurgery, and Psychiatry, 37, 10931098.

Leech, P., and Miller, J. D. (1974b). Intracranial volume-pressure relationships during experimental brain compression in primates. 2. Effect of induced changes in systemic arterial pressure and cerebral blood flow. Journal of Neurology, Neurosurgery, and Psychiatry, 37, 1099-1104.

Leyden, E. (1866). Beiträge und Untersuchungen zur Physiologie und Pathologie des Gehirns. Uber Hirndruck und Hirnbewegungen. Virchows Archiv für Pathologische Anatomie und Physiologie, 37, 519 559.

Lim, S. T., Potts, D. G., Deonarine, V., and Deck, M. D. F. (1973). Ventricular compliance in dogs with and without aqueductal obstruction. Journal of Neurosurgery, 39, 463-473.

Löfgren, J. (1973). Effects of variations in arterial pressure and arterial carbon dioxide tension on the cerebrospinal fluid pressure-volume relationships. Acta Neurologica Scandinavica, 49, 586-598.

Löfgren, J., and Zwetnow, N. N. (1973). Cranial and spinal components of the cerebrospinal fluid pressure-volume relationships. Acta Neurologica Scandinavica, 49, 586-598.

Löfgren, J., Essen, C. von, and Zwetnow, N. N. (1973). The pressure-volume curve of the cerebrospinal fluid space in dogs. Acta Neurologica Scandinavica, 49, 557-574.

Lundberg, N. (1960). Continuous recording and control of ventricular fluid pressure in neurosurgical practice. Acta Psychiatrica et Neurologica Scandinavica, 36, Supplement, 149.

Lundberg, N., Cronqvist, S., and Kjällquist, A. (1968). Clinical investigations on interrelations between intracranial pressure and intracranial haemodynamics. Progress in Brain Research, 30, 69-75.

Marmarou, A. (1973). A theoretical and experimental evaluation of the cerebrospinal fluid system. PhD thesis, Drexel University.

Marmarou, A., Shulman, K., and LaMorgese, J. (1975). Compartmental analysis of compliance and outflow resistance of the cerebrospinal fluid system. Journal of Neurosurgery, 43, 523-534.

Martins, A. N., Wiley, J. K., and Myers, P. W. (1972). 
Dynamics of the cerebrospinal fluid and the spinal dura mater. Journal of Neurology, Neurosurgery, and Psychiatry, 35, 468-473.

Miller, J. D., and Garibi, J. (1972). Intracranial volume-pressure relationships during continuous monitoring of ventricular fluid pressure. In Intracranial Pressure, pp. 270-274. Edited by M. Brock and H. Dietz. Springer: Berlin.

Miller, J. D., Garibi, J., and Pickard, J. D. (1973). Induced changes of cerebrospinal fluid volume. Effects during continuous monitoring of ventricular fluid pressure. Archives of Neurology (Chicago), 28, 265-269.

Miller, J. D., and Pickard, J. D. (1974). Intracranial volume-pressure studies in patients with head injury. Injury, 5, 265-268.

Mosso, A. (1881). Uber den Kreislauf des Blutes im menslichen Gehirn. Leipzig.

Nornes, H., Aaslid, R., and Lindegaard, K. F. (1977). Intracranial pulse pressure dynamics in patients with intracranial hypertension. Acta Neurochirurgica, 38, 177-186.

Ryder, H. W., Epsey, F. F., Kimbell, F. D., Penka, E. J., Rosenauer, A., Podolsky, B., and Evans, J. P. (1953). The mechanism of the change in cerebrospinal fluid pressure following an induced change in the volume of the fluid space. Journal of Laboratory and Clinical Medicine, 41, 428-435.

Severinghaus, J. W. (1966). Blood gas calculator. Journal of Applied Physiology, 21, 1108-1116.

Shapiro, H. M., Langfitt, T. W., and Weinstein, J. D.
(1966). Compression of cerebral vessels by intracranial hypertension. II Morphological evidence for collapse of vessels. Acta Neurochirurgica, 15, 223233.

Shulman, K., and Marmarou, A. (1971). Pressurevolume considerations in infantile hydrocephalus. Developmental Medicine and Child Neurology, 13, Supplement 25, 90-95.

Sullivan, H. G., Miller, J. D., Becker, D. P., Flora, R. E. and Allen, G. (1977). The physiological basis of intracranial pressure change with progressive epidural brain compression. An experimental $\stackrel{\vec{s}}{\rho}$ evaluation in cats. Journal of Neurosurgery, 47, 532-550.

Symon, L., Pasztor, E., Branston, N. M., and Dorsch, N. W. C. (1974). Effect of supratentorial spaceoccupying lesions on regional intracranial pressure $\stackrel{\mathbb{\Omega}}{\alpha}$ and local cerebral blood flow: an experimental study in baboons. Journal of Neurology, Neurosurgery, and Psychiatry, 37, 617-626.

Symon, L., Crockard, H. A., and Juhasz, J. (1975). Some aspects of cerebrovascular resistance in raised intracranial pressure: an experimental study. In Intracranial Pressure II, pp. 257-262. Edited by N. Lundberg, U. Pontén, and M. Brock. Springer: Berlin.

Szewczykowski, J., Sliwka, S., Kunicki, A., Dytko, PD and Korsak-Sliwka, J. (1977). A fast method of $\infty$ estimating the elastance of the intracranial systeno A practical application in neurosurgery. Journal of Neurosurgery, 47, 19-26. 